\title{
Recomendaciones metodológicas para los censos de lobos marinos comunes, Otaria flavescens
}

\author{
M. Florencia Grandi ${ }^{\varpi}$; Néstor A. García \& Enrique A. Crespo \\ Laboratorio de Mamíferos Marinos, Centro para el Estudio de Sistemas Marinos, CONICET.
}

\begin{abstract}
Resumen. Estimar y monitorear las tendencias poblacionales es esencial para comprender el proceso de recuperación y la dinámica poblacional de especies que se han visto afectadas por acción del hombre o que necesitan ser manejadas. Cuando se realizan censos poblacionales por períodos prolongados, y en especial si la especie en consideración tiene rangos de distribución amplios, esos monitoreos los realizan distintos grupos de trabajo a lo largo del tiempo y con diversos métodos. El objetivo de este trabajo es presentar recomendaciones metodológicas para llevar a cabo censos y estimaciones de abundancia poblacionales de Otaria flavescens a lo largo de su distribución. Nuestra meta es que estas recomendaciones se difundan entre todos los actores que monitorean las poblaciones de esta especie y que permitan sistematizar y estandarizar la toma de datos. Así, el esfuerzo realizado será comparable entre zonas y se podrá utilizar en el largo plazo para mejorar el conocimiento y la tendencia de las poblaciones en recuperación.
\end{abstract}

[Palabras clave: estimación de abundancia, clases de edad, Otaria flavescens, lobo marino sudamericano]

\begin{abstract}
Aвstract. Methodological recommendations for surveys of common sea lions, Otaria flavescens. The estimation and monitoring of population trends are essential to understand the recovery and population dynamics of species that have been affected by humans. When population censuses are carried out for long periods of time, and especially if the species under consideration has wide distributional ranges, different working groups with different methods make these surveys. The aim of this work is to present methodological recommendations to carry out population censuses and abundance estimates of Otaria flavescens throughout its distribution. Our ultimate goal is that the present work will allow to systematize and standardize data gathering. In this way, the effort made will be comparable between areas and can be used in the long term to improve the knowledge and trend of recovering populations.
\end{abstract}

\section{INTRODUCCIÓN}

Durante los últimos dos siglos ha tenido lugar una reducción general en la abundancia y la distribución espacial de muchas especies marinas (Costello et al. 2010). En este contexto, monitorear y estimar las tendencias de la abundancia y la distribución geográfica es esencial para comprender el proceso de recuperación y la dinámica poblacional de las especies que se vieron afectadas, así como para manejar y conservar las poblaciones animales (Anganuzzi 1993; Caughley and Gunn 1996; Forney 2000); especialmente, de aquellas especies marinas de gran tamaño (Payne et al. 2016).

El lobo marino común o de un pelo, Otaria flavescens (Shaw 1800), es una especie endémica de América del Sur. Se distribuye a lo largo de todo el litoral marítimo de Sudamérica, desde Torres [Brasil $\left(29^{\circ} \mathrm{S}\right)$ ] hasta Tierra del Fuego en el Océano Atlántico (incluyendo las
Islas Malvinas) y desde el Cabo de Hornos [Chile] hasta Zorritos [Perú $\left(4^{\circ} \mathrm{S}\right)$ ], sobre el Océano Pacífico (Riedman 1990; Rosas et al. 1994) (Figura 1). Los lobos marinos se agrupan en numerosas colonias (apostaderos o paraderos) continentales e insulares (Figura $1)$, con desplazamientos entre las mismas (Ximénez 1975; Lewis and Ximénez 1983; Crespo 1988; Sepúlveda et al. 2015; Giardino et al. 2016). Esta especie fue intensamente explotada comercialmente durante el siglo XX (Carrara 1952; Godoy 1963; Páez 2006) y se encuentra en recuperación. Sin embargo, es probable que la abundancia actual ( 445000 individuos) (Cárdenas-Alayza et al. 2016) esté muy por debajo de las cifras anteriores de la explotación, con base en lo que ocurre en la población del Atlántico, que actualmente representa un tercio del tamaño original que tuvo antes de la explotación, y es la única en la que se estimó retrospectivamente su tamaño original (Crespo 2021). 


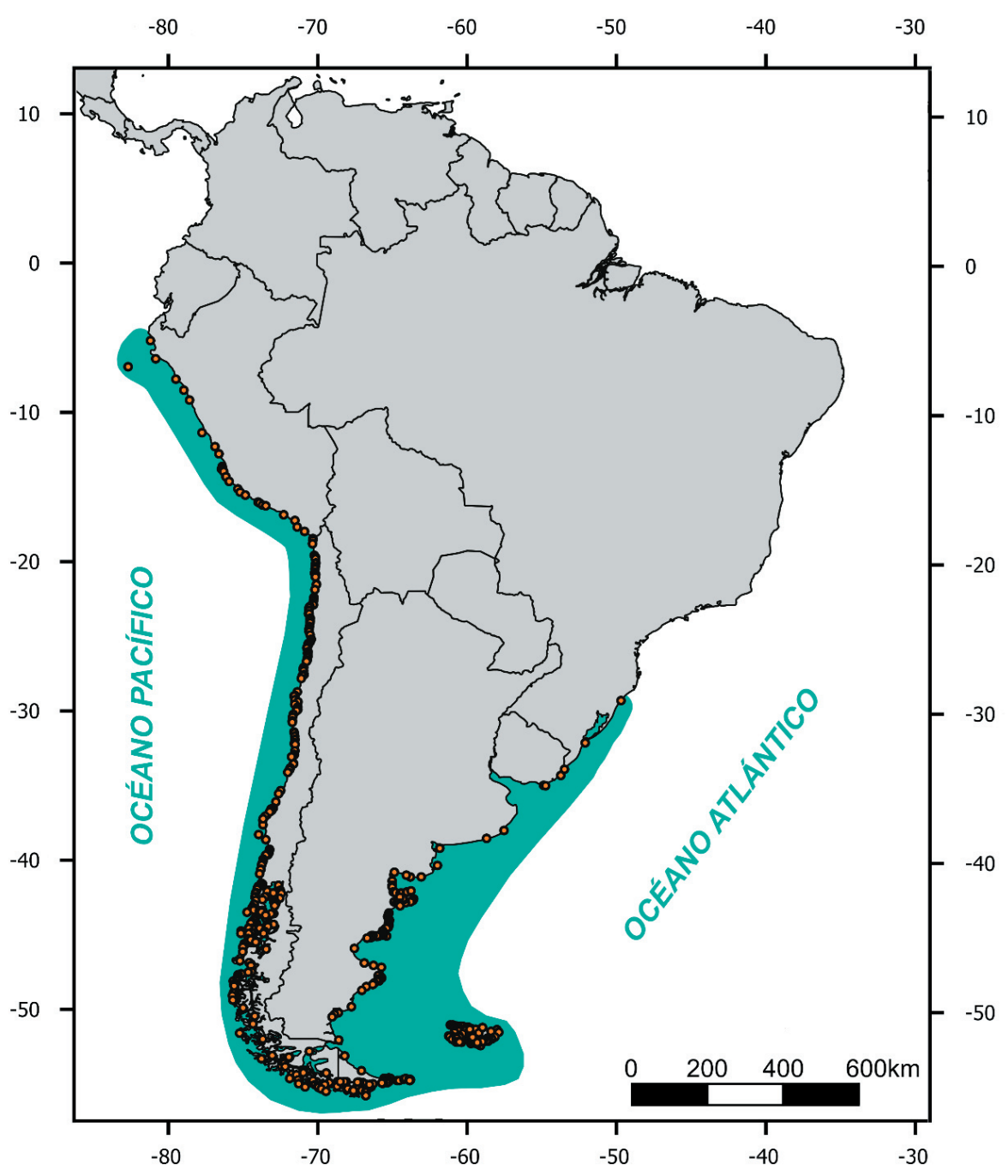

Figura 1. Distribución geográfica del lobo marino común, Otaria flavescens, indicando con puntos sus apostaderos en Sudamérica.

Figure 1. Geographic distribution of South American sea lion, Otaria flavescens, indicating their colonies with dots.

Estudios genéticos recientes indican que las agrupaciones de $O$. flavescens de los océanos Atlántico y Pacífico son diferentes, por lo que se deben considerar como unidades evolutivamente significativas distintas (Oliveira et al. 2017), a pesar de que existe cierto intercambio o flujo de individuos entre ellas (Weinberger 2013; Hoffman et al. 2016; Oliveira et al. 2017). Además, dentro de las grandes áreas de reproducción del Atlántico (Uruguay, distintas zonas de la Patagonia e Islas Malvinas), varios estudios sugieren un alto flujo de genes mediado por los machos y un bajo flujo génico de las hembras, lo que genera distintas unidades poblacionales o stocks (Túnez et al. 2007, 2010; Feijoo et al.
2011; Hoffman et al. 2016; Oliveira et al. 2017; Grandi et al. 2018).

La recuperación de las poblaciones de $O$. flavescens en los océanos Pacífico y Atlántico Sur ha sido muy variable entre zonas. Los stocks de Uruguay y los del extremo austral de Chile están disminuyendo (Venegas et al. 2002; Franco-Trecu et al. 2015). El stock del centro de Chile es estable (Sepúlveda et al. 2011), los de las Islas Malvinas, los de Perú y los del norte y del sur de Chile se recuperan lentamente (Bartheld et al. 2008; Oliveira and Majluf 2012; Oliva et al. 2012, 2020; Contreras et al. 2014; Baylis et al. 2015), mientras que los stocks de la costa atlántica patagónica están aumentando 
rápidamente (Reyes et al. 1999; Grandi et al. 2008, 2015; Milano et al. 2020). Sin embargo, todos los stocks todavía están expuestos a una variedad de impactos negativos reales (i.e., interacciones operacionales con la salmonicultura y diversos tipos de pesquerías, contaminación) y potenciales (i.e., prospección sísmica, rutas navieras) relacionados con el desarrollo de las actividades humanas costeras (Bastida and Rodríguez 2003; Crespo et al. 2012, 2021; Consejo Federal Pesquero 2016; Cárdenas-Alayza et al. 2016; Cárdenas-Alayza 2018). Los efectos de las interacciones ecológicas indirectas son aun más sutiles debido al consumo que hacen los lobos marinos de especies capturadas por la pesca en gran escala, tanto comerciales como descartadas (Crespo 2021).

Elmonitoreoy las estimaciones deabundancia de las poblaciones de O. flavescens, o de sus colonias, resulta indispensable para conocer el estado de las mismas, sus tendencias y sus cambios a lo largo del tiempo. La estimación de la abundancia incluye varias técnicas metodológicas para estimar el tamaño de una población (i.e., su abundancia absoluta), o índices de abundancia relativa proporcionales al tamaño de la población, lo que permite evaluar las tendencias de la población en el espacio y en el tiempo (Buckland and York 2018). Las técnicas principales para estimar la abundancia (absoluta o relativa) de pinnípedos son el marcado-recaptura y el conteo del total de individuos presentes en las colonias o una estimación del mismo (Eberhardt et al. 1979; Buckland and York 2018).

La especie en consideración tiene amplios rangos de distribución. Por lo tanto, esos monitoreos son realizados por distintos grupos de trabajo (investigadores, guardafaunas o guardaparques de reservas o áreas protegidas o personal y voluntarios de ONG). Incluso, a lo largo del tiempo, los miembros de esos grupos de trabajo van cambiando y reemplazándose. Por ello es necesario sistematizar y estandarizar la toma de datos para que todo ese esfuerzo sea comparable entre zonas y colonias, y se lo pueda usar a largo plazo para mejorar el conocimiento, el estado y, lo más importante, la tendencia de las poblaciones o stocks. Por lo tanto, el objetivo de este trabajo consiste en exponer recomendaciones metodológicas para realizar los censos y estimaciones poblacionales de Otaria flavescens a lo largo de su rango de distribución. Esperamos que el mismo sea difundido e implementado entre todos los actores que realizan el monitoreo poblacional de esta especie.

\section{Materiales y Métodos}

Para concretar el objetivo propuesto se realizó una revisión detallada de la literatura disponible, así como la recopilación de más de 50 años de experiencia propia de trabajo de campo en la Argentina y de colegas latinoamericanos.

\section{Resultados y Discusión}

\section{Acceso a los apostaderos}

A lo largo de la costa, los apostaderos pueden encontrase incluidos dentro de un sistema de áreas protegidas o en la costa de propiedades privadas. Esto hace que para acceder sea necesario solicitar permisos de ingreso y de trabajo. Para el caso de las colonias que están en áreas protegidas, previo a la realización de los censos se debe cumplir con los requisitos administrativos de permisos de trabajo y considerar el tiempo que demora este trámite. Para el caso de las colonias en costas de privados se deben contactar a los propietarios y conversar para coordinar el ingreso. Teniendo en consideración lo anterior, se recomienda realizar estas diligencias administrativas con varios meses de anticipación a las campañas de trabajo de campo.

\section{Fecha del censo}

Para obtener una estimación poblacional lo más representativa posible, lo más conveniente es realizar el censo de los animales en la época reproductiva, que corresponde al período de mayor concentración de los animales en tierra. Teniendo en consideración que en distintas latitudes puede haber un desfase de días en la fecha de los eventos reproductivos en Otaria flavescens (Acevedo et al. 2003; Pavés et al. 2005), para el caso de las colonias del Atlántico y las del centro y sur de Chile, se recomienda realizar el censo en el transcurso de la última semana de enero, debido a que en ese período la mayoría de los machos y hembras adultas no abandonan las colonias para realizar viajes de alimentación, y ya ha nacido más del 80\% de las crías (Figura 2A) (Campagna 1985; Crespo 1988; Pavés et al. 2005). Para el caso de las colonias del norte del Océano Pacífico se recomienda realizar el censo en la primera semana de febrero (Figura 


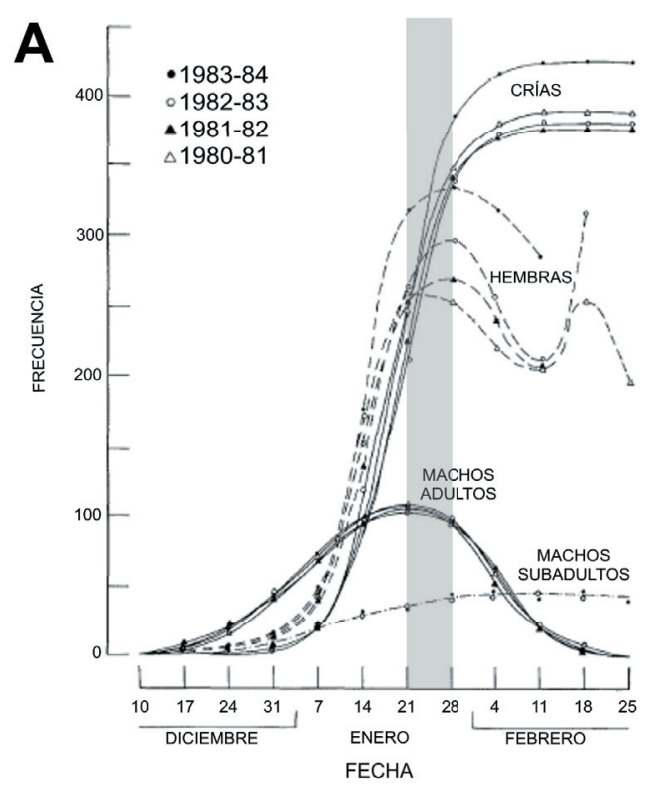

Figura 2. Número promedio de machos, hembras y crías. A: durante cuatro temporadas reproductivas en el apostadero de Punta Norte, Chubut, Argentina (imagen extraída de Campagna 1985); B: durante dos temporadas reproductivas en el apostadero de Punta Negra, I Región, Chile (imagen extraída de Acevedo et al. 2003). En sombreado gris se indica el período de mayor concentración de animales en tierra, recomendado para realizar los censos de estimación poblacional.

Figure 2. Average number of males, females and pups. A: during four reproductive seasons at Punta Norte colony, Chubut, Argentina (image from Campagna 1985); B: during two reproductive seasons at Punta Negra colony, Region I, Chile (image from Acevedo et al. 2003). Grey rectangles indicate the period with the highest concentration of animals on land, recommended for conducting population censuses.

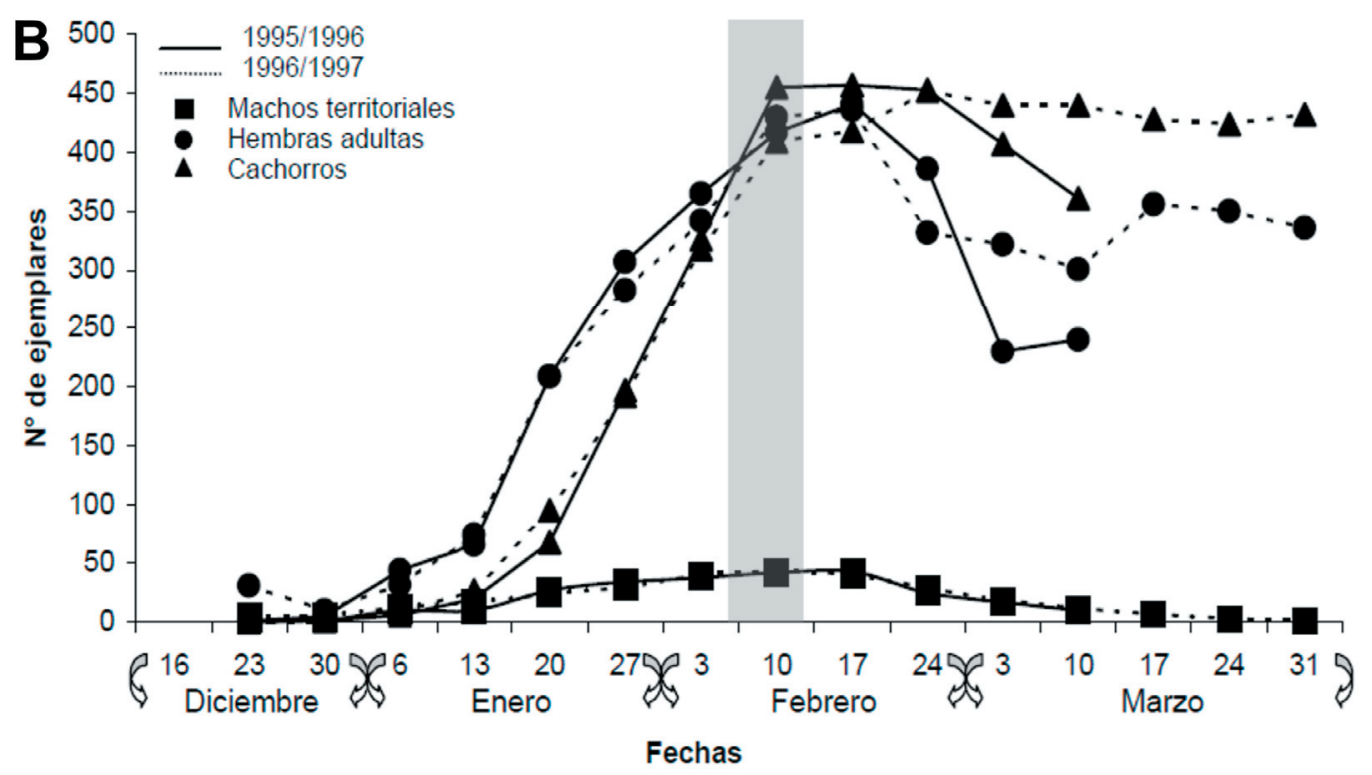

2B). Si por motivos climáticos o logísticos no es posible realizar el censo en el período de mayor concentración de animales, luego se deberán ajustar los conteos aplicando factores de corrección que dependen de la presencia de cada clase de edad en las distintas semanas (Crespo 1988; Sepúlveda et al. 2012).

Si el censo se realiza a fines de la temporada de cría (i.e., luego de los últimos nacimientos), se puede obtener una estimación de la cantidad de crías nacidas (i.e., la productividad del apostadero), pero se subestimarán las demás clases de edad. Si el objetivo del censo es otro [e.g., estimar la presencia y la variabilidad de los animales en un apostadero a lo largo del año, la abundancia de individuos fuera de la temporada reproductiva, analizar apostaderos no reproductivos o estudios de ritmos circadianos o estacionales (Sepúlveda et al. 2001, 2009, 2012, 2015; Mandiola et al. 2010; Pavanato et al. 2013; Giardino 2014)], la fecha del censo se puede ajustar para responder esas otras interrogantes, y la metodología del conteo se mantiene.

\section{Hora del día y altura de la marea}

Además de la variación en la abundancia de animales en un sitio a lo largo del año, 
la abundancia de una colonia está sujeta a cambios relacionados con el comportamiento circadiano de la especie, asociado a actividades de alimentación (Sepúlveda et al. 2001, 2012, 2015). Para O. flavescens se describió que la abundancia en las colonias disminuye hacia el atardecer, aumenta al amanecer y es máxima al mediodía y en las primeras horas de la tarde ( 14:00 h) (Rosas et al. 1994; Sepúlveda et al. 2001, 2012, 2015). Por lo tanto, es muy recomendable que los censos se efectúen en el período de horas comprendido entre el mediodía y la media-tarde (i.e., desde las 12: 00 a las 18:00).

Otros factores a tener en consideración en los conteos se relacionan con la fase lunar, el ciclo de mareas y su altura en los apostaderos (Reyes and Crespo 1994). La diferencia en la altura de las mareas aumenta con la latitud, lo cual hace que esta variable sea relevante cuanto más austral es el apostadero. El efecto de la altura de la marea sobre la disposición y abundancia de los lobos en una colonia va a ser mayor en sitios donde la pendiente de la costa es pronunciada, por lo que se recomienda realizar el censo durante la bajamar. Además, a lo largo del ciclo lunar hay variaciones en el número de animales en la costa: es mayor en luna llena y cuarto creciente y menor en luna nueva y cuarto menguante. El ritmo lunar estaría vinculado a variaciones en la distribución vertical de las presas, acorde a la intensidad de la luz lunar en la columna de agua (Reyes and Crespo 1994). Para el caso que se quieran aplicar factores de corrección es necesario que se realicen estudios auxiliares a fin de calcular esos factores durante períodos y lugares representativos del área donde se realiza el censo principal (Bartheld et al. 2008; Contreras et al. 2014).

\section{Registro de datos y fotografías}

Antes del censo se deberán obtener fotografías panorámicas y, en la libreta de campo, realizar un croquis de la costa con la ubicación de todos los grupos, designándose números o letras a cada uno de los grupos diferenciados para luego contar el número de animales. Se debe agregar al croquis los mayores detalles posibles (e.g., características topográficas como cañadones, caletas, acantilados, restingas y lagunas, y otras como alambrados, cascos de estancias, faros, balizas, etc.) que ayuden en su ubicación en futuros monitoreos (Figura 3). También se debe registrar, con un GPS, la posición geográfica del observador o desde dónde se toman las fotografías. Si es posible, se recomienda registrar la posición geográfica de cada grupo para ubicarlos luego sobre un mapa.

Se deben realizar notas y observaciones de los factores ambientales antes y en el momento del censo, tales como la velocidad y la dirección del viento, el estado de nubosidad (i.e., despejado, parcialmente nublado, nublado), el porcentaje de resolana (i.e., brillo o resplandor en el mar), la presencia/ausencia de lluvia/ neblina, el estado y la altura del mar, etc. También se deben registrar otros factores en las observaciones, como si hay alguna saliente del acantilado que impida el conteo de animales dentro de cuevas, etc. Estas anotaciones pueden luego ayudar a evaluar el censo (e.g.,

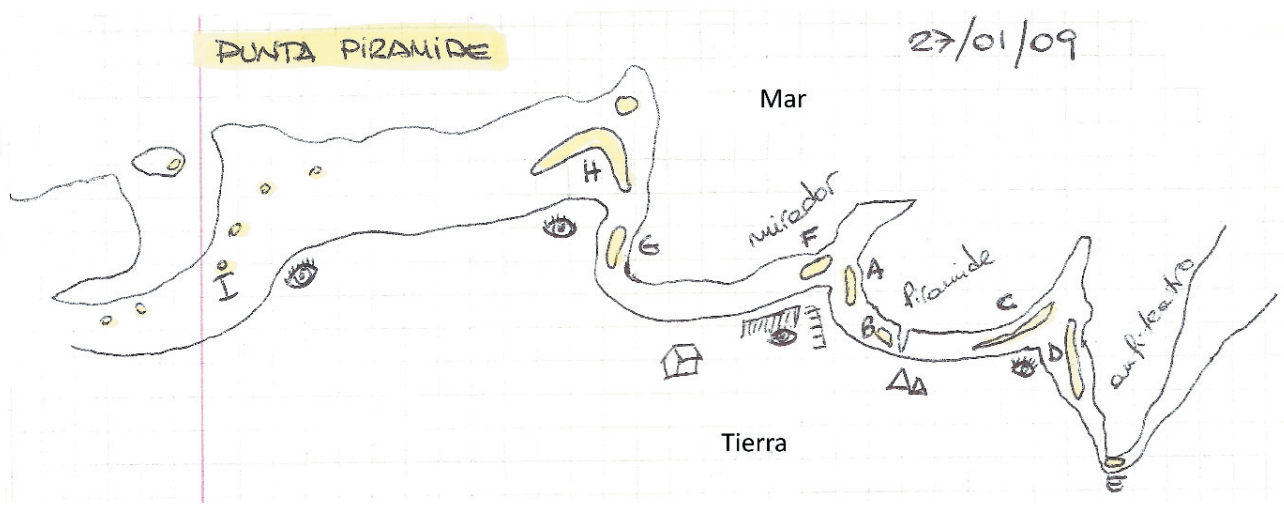

Figura 3. Ejemplo de croquis realizado en la colonia de Punta Pirámide durante el censo 2009, esquematizando los grupos de lobos (amarillo y letras A-I), los puntos de observación (dibujo de ojos), características topográficas (restingas, puntas, bahías) y otras características (casa del guardafauna y barandas de los miradores).

Figure 3. Example of a sketch made in Punta Pirámide colony during the 2009 census, outlining the groups of sea lions (yellow and letters A-I), the observation points (drawing of eyes), topographic characteristics (restingas, points, bays) and other characteristics (house of the ranger and railings of the viewpoints). 
si el viento es muy fuerte, se dificulta el conteo con binoculares y entonces la estimación de abundancia puede resultar subestimada). Por otro lado, si al momento del censo se inicia una estampida de los animales al agua por efecto de la presencia de observadores, se deberá suspender el conteo, esperar un tiempo quieto en el lugar y reiniciar el conteo desde cero cuando los animales hayan retornado a tierra y se encuentren tranquilos. Además, se debe registrar la hora de inicio y finalización del censo. En caso de existir informes, censos o trabajo de campo previos en un sitio o área, se recomienda mantener la denominación utilizada en ellos o al menos hacer referencia, en un apartado de observaciones en la libreta de campo, si su ubicación coincide con los grupos o sitios censados.

\section{Conteos}

Censo terrestre. Se deberá buscar un punto elevado (plataforma artificial o natural) desde donde se tenga la mejor visibilidad de la colonia y de cada grupo. Si es necesario, el observador podrá moverse a lo largo de la colonia, deteniéndose frente a cada grupo donde mejor se vean todos los animales de ese grupo. Los conteos en cada apostadero deben realizarse sobre grupos de animales, empleando la metodología de conteos totales (Eberhardt et al. 1979), con binoculares (8x30 y 10x50) o a ojo desnudo. Lo anterior dependerá de la distancia y la altura entre el punto de observación y el grupo de animales. Usar un contador manual facilita el conteo. Se debe seleccionar un grupo de lobos marinos a censar (definiendo los límites del grupo de acuerdo con características topográficas o interrupciones de los agrupamientos) en el cual se realizarán dos o más conteos de cada clase de edad presente en ese grupo, hasta que la diferencia entre los conteos no exceda el 10\% del promedio entre conteos. De esta manera el error del observador es fijado a priori, y en situaciones óptimas (grupos $<30$ animales) es cercano a cero (Crespo 1988). Todos los conteos realizados por cada observador deben ser anotados en la libreta o planilla (Tabla 1) para luego estimar el error del conteo y el error entre observadores. La estimación final para cada clase de edad se obtiene promediando los valores de la serie que se encuentran dentro del margen de error previsto (Crespo 1988). Finalmente, la estimación total del apostadero corresponderá a la sumatoria de las estimaciones totales de cada grupo que lo conforma (Tabla 1).

Censo aéreo. Los relevamientos aéreos se pueden efectuar mediante un vuelo en aeronave tripulada (preferentemente bimotor de ala alta, por seguridad y visibilidad) o por medio de un dron (vehículos aéreos no tripulados), en los cuales el conteo se realiza sobre un mosaico de fotografías de la colonia. La altitud de vuelo apropiada para obtener fotografías aéreas en una aeronave tripulada varía entre 170 y $600 \mathrm{~m}$, y la velocidad de la aeronave debería ser mínima, dependiendo de la altura de la costa y del objetivo del lente (Schiavini et al. 2004; Wright 2005; Bartheld et al. 2008). En el caso de realizar el sobrevuelo de la colonia con una aeronave no tripulada

Tabla 1. Ejemplo de una planilla utilizada para el conteo de Otaria flavescens en el apostadero de Punta Pirámide en 2009. Los grupos se corresponden con aquellos graficados en el croquis de la Figura 3.

Table 1. Example of a spreadsheet used to count Otaria flavescens at Punta Pirámide colony in 2009. The groups correspond to those shown in Figure 3.

\begin{tabular}{|c|c|c|c|c|c|c|c|c|c|c|c|c|c|}
\hline $\begin{array}{l}\text { Aposta- } \\
\text { dero }\end{array}$ & Fecha & Hora & Marea & Grupo & $\mathrm{MH}$ & $\mathrm{M}$ & $\begin{array}{c}\text { MSA } \\
\text { II }\end{array}$ & $\begin{array}{c}\text { MSA } \\
\text { III }\end{array}$ & $\mathrm{H}$ & $\mathrm{J}$ & $\mathrm{H}+\mathrm{J}$ & $\mathrm{C}$ & Total \\
\hline $\begin{array}{l}\text { Pta. } \\
\text { Pirámide }\end{array}$ & $27 / 01 / 2009$ & $14: 00$ & Baja & A, Pirámide & 3 & 2 & 0 & 0 & $30 / 32 / 32$ & 0 & 0 & $33 / 34 / 34$ & 70 \\
\hline Pta. & $27 / 01 / 2009$ & $14: 20$ & Baja & B, Pirámide & 2 & 0 & 1 & 1 & 7 & 0 & 0 & 8 & 19 \\
\hline $\begin{array}{l}\text { Pta. } \\
\text { Pirámide }\end{array}$ & $27 / 01 / 2009$ & $14: 30$ & Baja & C, Pirámide & $8+9$ & 3 & 2 & 3 & $\begin{array}{c}76 / 78+ \\
67 / 68 / 69\end{array}$ & 1 & 0 & $\begin{aligned} 83 / 84 / 85 & +94 / 96 / 102 \\
& +6\end{aligned}$ & 358 \\
\hline & $27 / 01 / 2009$ & 15:02 & Baja & D, anfiteatro & $10+5$ & 3 & 2 & 3 & $150+26$ & 0 & 0 & $\begin{array}{l}177 / 174 / 178 \\
+21+52 / 53\end{array}$ & 449 \\
\hline $\begin{array}{l}\text { Pta. } \\
\text { Pirámide }\end{array}$ & $27 / 01 / 2009$ & $15: 25$ & Baja & E, anfiteatro & 5 & 1 & 3 & 1 & $9+29+2$ & 0 & 0 & $49 / 47 / 48$ & 98 \\
\hline $\begin{array}{l}\text { Pta. } \\
\text { Pirámide }\end{array}$ & $27 / 01 / 2009$ & $15: 35$ & Baja & F, mirador & 3 & 1 & 2 & 0 & 17 & 1 & 0 & $27 / 28$ & 52 \\
\hline $\begin{array}{l}\text { Pta. } \\
\text { Pirámide }\end{array}$ & $27 / 01 / 2009$ & $15: 47$ & Baja & G, restinga & 9 & 1 & 2 & 0 & $39 / 40 / 43$ & 0 & 0 & $59 / 60 / 58$ & 112 \\
\hline $\begin{array}{l}\text { Pta. } \\
\text { Pirámide }\end{array}$ & $27 / 01 / 2009$ & $15: 55$ & Baja & $\mathrm{H}$, restinga & $8+15$ & $1+5$ & $2+8$ & $2+13$ & $\begin{array}{c}78 / 77 / 81 / 78+ \\
81 / 78+31\end{array}$ & 0 & 0 & $\begin{array}{c}14+66 / 63 / 61+ \\
114 / 124 / 121+25\end{array}$ & 466 \\
\hline $\begin{array}{l}\text { Pta. } \\
\text { Pirámide }\end{array}$ & $27 / 01 / 2009$ & $16: 20$ & Baja & $\begin{array}{l}\text { I, roca barco } \\
\text { y restinga }\end{array}$ & 0 & 3 & 5 & 19 & 0 & 9 & 0 & $2+$ & 38 \\
\hline $\begin{array}{l}\text { Pta. } \\
\text { Pirámide }\end{array}$ & $27 / 01 / 2009$ & & & TOTAL & 77 & 20 & 27 & 42 & 647 & 11 & 0 & 838 & 1662 \\
\hline
\end{tabular}

t: indica ejemplares muertos vistos en el grupo.

Los números separados por ' $/$ ' indican distintos conteos hechos por un observador sobre el mismo grupo. 
(dron), se recomienda una altura mínima de $30 \mathrm{~m}$, y se deberá aumentar la altura en caso de haber colonias de aves marinas (Raoult et al. 2020). Las fotografías aéreas se deben realizar en forma secuencial y con una cierta superposición entre fotografías consecutivas. Ese mosaico debe ser construido seccionando y utilizando las mejores fotografías, en base a su resolución, enfoque y claridad (Figura 4). Luego, el conteo debe realizarse por distintos observadores utilizando el software OTARIIDAE $\mathrm{u}$ otro software libre [e.g., Image J, ImageJ2 o QGIS (QGIS Development Team 2019; Rasband 2013; Rueden et al. 2017)] que permita al observador colocar puntos de referencia (e.g., de diferentes colores) sobre cada individuo por categoría de edad, para el posterior recuento total de la imagen analizada (Bartheld et al. 2008).
Censo marítimo. El censo marítimo se lleva a cabo desde embarcaciones y es utilizado en zonas donde el acceso terrestre es complejo o inaccesible, o en zona de fiordos con cobertura de bosques que impiden la realización de censos aéreos (Bartheld et al. 2008; Sepúlveda et al. 2009, 2011; Contreras et al. 2014; Baylis et al. 2015). En este tipo de censos es recomendable tomar fotografías secuenciales desde el punto más alto de la embarcación; si no se tiene un punto elevado, entonces realizarlos durante la marea alta para minimizar la diferencia de altura o la distancia entre la embarcación y el apostadero. El procedimiento de conteo desde embarcaciones sigue los mismos pasos metodológicos del censo terrestre. Se debe considerar que este tipo de censos puede producir alguna subestimación de la abundancia (debido a que la altura de la embarcación no
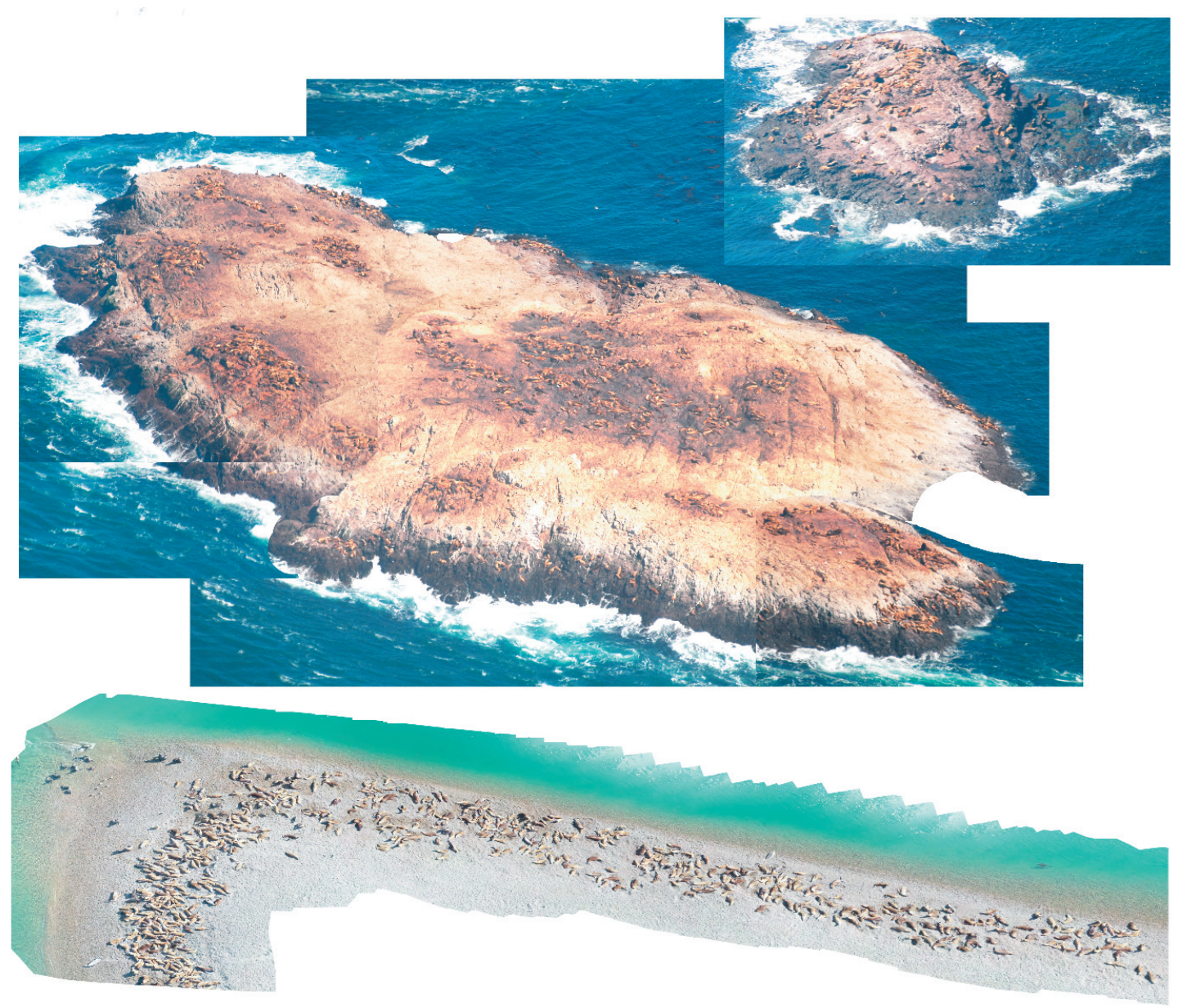

Figura 4. Ejemplo de dos mosaicos construidos a partir de fotos. Arriba: tomadas desde un avión de la colonia de Isla Blanca y su islote adyacente (Santa Cruz, Argentina). Abajo: tomadas desde un dron de la colonia de Caleta Valdés (Chubut, Argentina).

Figure 4. Example of two orthomosaics constructed from photos. Above: taken from a plane at Isla Blanca colony and its adjacent islet (Santa Cruz, Argentina). Below: taken from a drone at Caleta Valdés colony (Chubut, Argentina). 
permite obtener buenas fotografías y algunos de los animales quedan ocultos tras otros [ver Reyes et al. 1999; Bartheld et al. 2008; Sepúlveda et al. 2009]). Sin embargo, en muchas ocasiones, este tipo de censo resulta indispensable para completar la estimación poblacional o es el único medio de acceso posible a los apostaderos localizados en zonas inaccesibles desde tierra o aire.

En el caso de los pinnípedos, todos los métodos de estimación poblacional tienen sus ventajas y desventajas (Aragones et al. 1997; Buckland and York 2018; Raoult et al. 2020). El censo terrestre puede ser un método económico para obtener información cuantitativa sobre las tendencias en la abundancia de algunas poblaciones, pero requiere un amplio apoyo logístico si el área a cubrir es extensa, y en colonias densamente pobladas el conteo se dificulta (Udevitz et al. 2005). El método de captura-marca-recaptura es muy costoso y laborioso, requiere de un gran apoyo logístico y personal capacitado, el mantenimiento a largo plazo de los muestreos y las estimaciones son sensibles ante la falla de los supuestos implícitos para la estimación de abundancia (Buckland and York 2018). Por dichas razones no hemos desarrollado esta metodología. Los censos aéreos se pueden utilizar para obtener información sobre la distribución y la abundancia en grandes áreas geográficas en poco tiempo; estos estudios son menos costosos que los de marcación, más costosos que los censos terrestres y subestiman el conteo de crías (Aragones et al. 1997; Buckland and York 2018; Raoult et al. 2020). En efecto, algunos estudios detectaron diferencias importantes en exactitud y precisión al comparar conteos terrestres y aéreos (Lowry 1999; Bartheld et al. 2008; Franco-Trecu et al. 2019). Por último, usar drones para estimar las abundancias de poblaciones de pinnípedos (Raoult et al. 2020) permite obtener fotografías de mejor resolución que los censos con aviones y mejora el conteo de crías, en comparación con los conteos terrestres o desde aviones (Johnston et al. 2017; McIntosh et al. 2018; Rueda et al. 2020). Sin embargo, tienen desventajas que se deben tener en cuenta, como el tiempo de duración de las baterías, la capacidad de memoria para almacenar imágenes a campo y el tiempo de procesamiento de las mismas hasta obtener la estimación final (Raoult et al. 2020).

El tiempo total empleado en realizar un censo que abarca un área geográfica extensa debe ser el menor posible para disminuir el sesgo que pueda haber por el movimiento de individuos entre colonias cercanas. En el caso de un censo aéreo, este tiempo es menor y además puede realizarse con una menor variabilidad de las horas del día empleadas para tomar las fotografías. Sin embargo, los censos terrestres no sólo tienen la ventaja de obtener datos más detallados acerca de la composición social, sino que se realizan con mucho menor costo económico. Por otro lado, los censos con dron también disminuyen el tiempo empleado en el trabajo de campo pero, si el área geográfica es extensa, hay que organizar una logística de trasladados entre sitios para poder despegar el dispositivo. Además, siempre que se realice un relevamiento aéreo (ya sea con avión o dron) es recomendable contar con un relevamiento terrestre simultáneo de alguno de los sitios para poder construir factores de corrección de posibles sesgos de observación (e.g., Reyes et al. 1999; Grandi 2010; Bartheld et al. 2008; Contreras et al. 2014; Grandi et al. 2015; Milano et al. 2020) y validar las clases de edad y la composición social de las colonias.

\section{Tipos de apostaderos}

Los asentamientos de lobos marinos pueden clasificarse como permanentes (anuales o estables) o estacionales (transitorias o temporales) (Carrara 1952). Además, según el tipo de actividad realizada en las colonias, se pueden definir como de cría o reproductivas (apostaderos reproductores o parideros) o noreproductivas (también llamados apostaderos de descanso) (Carrara 1952). Esta clasificación define un proceso dinámico de las colonias, ya que en los últimos años se observaron cambios espacio-temporales en la ubicación y la composición de los apostaderos, ligados al aumento poblacional (Dans et al. 2004; Grandi et al. 2008, 2015). Estos cambios incluyen la ocupación espacial de nuevas áreas, el desarrollo de áreas nuevas de cría en apostaderos no-reproductivos y la transformación de la estructura social en el transcurso del tiempo (Crespo 1988, 2021; Crespo and Pedraza 1991; Grandi et al. 2008, 2015). Esto hace que los apostaderos reproductores puedan diferenciarse, de acuerdo con su estructura social, en apostaderos tradicionales (aquellos con una proporción importante de crías, 20-50\%) y apostaderos mixtos (aquellos que presentan una proporción importante de juveniles y machos subadultos, y una pequeña proporción de crías, <20\%) (Reyes et al. 1999; Dans et al. 2004; Grandi et al. 2008, 2015; Milano et al. 2020). 


\section{Clases de edad y sexo}

Si es posible, en los censos, los ejemplares de Otaria flavescens deben ser diferenciados en las siguientes clases de edad y sexo: machos adultos (MAD), machos subadultos (MSA), hembras adultas $(\mathrm{H})$, juveniles de ambos sexos $(\mathrm{J})$, crías $(\mathrm{C})$ y hembras+juveniles $(\mathrm{H}+\mathrm{J})$. Esta última categoría se utiliza para contar grupos grandes de individuos a los cuales no se les puede asignar ni el sexo, ni la clase de edad. Generalmente, esta categoría se emplea en el caso de apostaderos mixtos donde los juveniles están junto con grupos de hembras adultas, debido a que son muy similares.

En temporada reproductiva, los machos adultos se pueden identificar como machos con hembras $(\mathrm{MH})$ y machos periféricos o solitarios (M), y los machos subadultos pueden distinguirse también en 2 categorías: MSA II y MSA III. Durante el período no reproductivo se utilizan las categorías macho adulto y subadultos (II y III). Estas categorías se basan fundamentalmente en caracteres morfológicos externos, pero, además, por intermedio de patrones conductuales típicos de cada categoría desplegados durante la temporada de reproducción o post-reproducción y por su ubicación en la colonia (ver Material Suplementario). Estos caracteres son observables a simple vista o con binoculares (en campo o sobre fotografías) y, en general, no son definitorios por sí solos, sino que deben ser tenidos en cuenta en forma integrada (i.e., al momento de clasificar un individuo se observan su patrón conductual, caracteres externos y ubicación en la colonia, para incorporarlo en una de las clases de edad).

\section{Estimación del tamaño poblacional}

Los censos, incluso si se realizan durante la temporada reproductiva, representarán una estimación relativa de la abundancia poblacional porque subestiman el tamaño de la población; esto obedece a que una proporción desconocida de animales se encontrará en el mar en el momento del censo (Crespo 1988; Dans et al. 2004; Bedriñana-Romano et al. 2014; Buckland and York 2018). Por lo tanto, para estimar el tamaño total de la población (i.e., su abundancia absoluta) es necesario desarrollar y aplicar factores de corrección sobre el total de animales censados, de manera de incorporar los animales que no están en tierra al momento del censo. Lo ideal es que se desarrollen factores de corrección específicos para cada área geográfica considerando la época del año, el tipo de colonias, ritmo circadiano y su estructura social.

Para el caso de la Argentina, están disponibles factores de corrección que fueron desarrollados a partir de tablas de vida para la población de la Patagonia norte (Argentina). Estos factores utilizan la sumatoria de los valores de supervivencia y asumen una población estacionaria. Así, a partir de la relación total estimado/total censado se estimó un factor de corrección para los censos igual a 1.8 (Crespo 1988), lo que significa que $44.44 \%$ de los animales no estaría presente en tierra durante los censos en época reproductiva. Otra opción es la implementada por Grandi (2010), quien, utilizando los censos de la población de la Patagonia norte (Argentina) desde 1983 hasta 2009, aplicó una corrección a los valores de las distintas clases de edad según datos de presencia y movilidad obtenidos de bibliografía, considerandola distinta estructura social que tiene cada apostadero y asumiendo una población en crecimiento sostenido. Así, la regresión lineal entre el número de animales censados y el número de animales corregidos resultó en un factor de corrección para los censos igual a 1.90 (Grandi 2010). Este valor significa que $47.37 \%$ de los animales no estaría presente en tierra durante los censos. Una tercera aproximación para esta especie fue implementada por Romero et al. (2017), quienes, aplicando un modelo de crecimiento poblacional denso-dependiente, calcularon un coeficiente de detectabilidad $q$ (una constante de proporcionalidad que representa la fracción de la población observada en los censos) que varía entre 0.547 y 0.563 dependiendo del valor de capturas incidentales por pesca que se considere que sufre la población (Romero et al. 2017). Estos coeficientes implican que entre $43.7 \%$ y $45.3 \%$ de los animales no estaría presente en tierra durante los censos, lo que corresponde a un factor de corrección para los censos igual a 1.78 y 1.83, respectivamente.

En Chile se han aplicado diversos factores de corrección a los censos: por los indeterminados, por la fecha de nacimiento de las crías, por el tipo de censo (marítimo o aéreo), por la proporción hembras:crías y por la fecha y hora del día en que se realizó el censo (e.g., Sepúlveda et al. 2001, 2011, 2012, 2015; Oliva et al. 2008, 2012; Contreras et al. 2014). Otro enfoque fue obtener una estimación directa de la abundancia de animales en el mar, realizada mediante muestreos de transectas lineales (Buckland et al. 2001), que sugiere que 
al sur de Chile, al menos 20\% de la población se encuentra en el agua al momento del censo a fines de la temporada reproductiva (Bedriñana-Romano et al. 2014), y refuerza la propuesta de realizar censos simultáneos en tierra y sobre el mar para lograr evaluar el tamaño real de la población (BedriñanaRomano et al. 2014).

Existen otros métodos indirectos disponibles para estimar la abundancia total de la población (Garner et al. 1999). Uno es censar una parte de la población de un área geográfica. La principal ventaja de este método es que representa un costo logístico y económico sustancialmente menor al de un censo total. No obstante, para que sea válido es necesario que las colonias seleccionadas representen una proporción relevante de la población total y que esta proporción se mantenga en el tiempo (Garner et al. 1999). En diversas poblaciones de pinnípedos se ha aplicado el método indirecto del conteo de crías para estimar el tamaño completo de una población. La principal ventaja de este método es que el censo de las crías ofrece un menor error de conteo debido a que las crías son fácilmente distinguibles del resto de las clases de edad y se encuentran todas en tierra al final de la temporada reproductiva. Es por ello que, para varias especies de pinnípedos, se toma la estimación relativa de su abundancia como un índice confiable del tamaño poblacional (Payne 1978; Berkson and Demaster 1985; Wickens and Shelton 1992; Lonergan et al. 2011). Sin embargo, se deben tener ciertas precauciones al momento de interpretar las tendencias en el número de crías y su relación con las tendencias de la población (Wickens and Shelton 1992).

\section{Estimación de la tendencia poblacional}

En ciertas oportunidades sólo se requiere conocer si la abundancia de la población aumenta, disminuyeosemantieneestacionaria. En estos casos, la tasa intrínseca de incremento poblacional (r) puede ser estimada a partir de la pendiente de una recta de regresión lineal de los valores del logaritmo natural del número de individuos (crías o totales) en función del tiempo (Caughley 1977). Cuantos más datos (temporadas de censos) se tenga, tanto mejor se podrá ajustar la estimación. La significancia se puede estimar mediante un análisis de varianza (ANOVA) de la regresión (York 1987). Esta tasa puede tomar valores positivos, negativos o cero; por lo tanto, la población crece si $r>0$, se mantiene estable si $r=0$, o decrece si $r<0$. Para realizar comparaciones entre años y entre poblaciones, esta tasa de cambio debe ser expresada como una tasa promedio anual de cambio de la población como $(\lambda-1)^{*} 100$, donde $\lambda$ es la tasa finita de incremento poblacional $\left(=e^{r}\right)$ (Caughley 1977).

\section{Conclusiones y RECOMENDACIONES}

Los programas de conservación y manejo de mamíferos marinos requieren datos precisos sobre la abundancia y la tendencia de la población (Teilmann et al. 2010). La metodología más adecuada para estas estimaciones dependerá de los objetivos del trabajo, la escala espacial, la ubicación, el presupuesto, el momento y la disponibilidad de apoyo logístico para llevarlo adelante (Aragones et al. 1997), sobre todo si se considera realizar un monitoreo a largo plazo. Habiendo discutido las ventajas y desventajas de los métodos de estimación poblacional, al momento de planificar los censos de pinnípedos es recomendable evaluar el área geográfica a relevar con las características particulares de su costa, las posibilidades de acceso a los apostaderos y la disponibilidad de financiamiento y de personal capacitado. Sobre la base de la experiencia previa y de varios estudios realizados en el lobo marino común o de un pelo (i.e., Crespo 1988; Reyes et al. 1999; Dans et al. 2004; Bartheld et al. 2008; Oliva et al. 2008; Sepúlveda et al. 2011, 2012; Contreras et al. 2014; Grandi et al. 2008, 2015) se recomienda realizar censos aéreos cada 5 años, de manera de registrar la aparición y la desaparición de nuevas colonias en un área geográfica extensa, y alternar con censos terrestres, marítimos o con dron más frecuentes (cada 2-3 años) de todas o algunas colonias, para así registrar información detallada sobre la composición social de las mismas y sus cambios en el tiempo. Durante los censos también es recomendable realizar encuestas a lugareños sobre los cambios en la distribución de las colonias o la aparición de grupos nuevos para su posterior monitoreo.

En caso de que se disponga de poco tiempo o de escasos recursos económicos, se recomienda al menos intentar mantener el monitoreo censando sólo las crías nacidas en temporada reproductiva de los apostaderos de cría. Este valor se podría utilizar como una estimación relativa de la abundancia poblacional. Si se cuenta con estimaciones previas, también se podrá calcular una tendencia poblacional en base al número de crías. 
Finalmente, para estimar la abundancia absoluta de la población a partir de un censo (considerando los individuos que se encuentran en el agua al momento del censo) se deben aplicar ajustes por las horas del día y época de la temporada o año, en caso de que cuente con seguimientos detallados en colonias particulares (e.g., Sepúlveda et al. 2001, 2011, 2012, 2015; Oliva et al. 2008; Contreras et al. 2014). Ante la falta de seguimientos detallados para desarrollar un factor de corrección propio del área se puede utilizar el factor de corrección más conservador de los conocidos a la fecha, como el factor de corrección de 1.8 (Crespo 1988). De todas formas, de ser posible, se recomienda construir otros factores de corrección en base a nuevos estudios en cada zona geográfica con sus características particulares y sobre la base de estos factores con los descriptos previamente.

Agradecimientos. A todos los compañeros del Laboratorio de Mamíferos Marinos
(CESIMAR, CONICET), y del Centro de Investigación Aplicada y Transferencia Tecnológica en Recursos Marinos Almirante Storni (CIMAS, CONICET) que a lo largo de los años acompañaron y realizaron las campañas de censos de lobos marinos en Patagonia Argentina. A los guardafaunas y guardaparques que compartieron con nosotros el trabajo de campo y que, con su vocación por conocer y conservar esta especie, insistieron en realizar este trabajo. A tres revisores anónimos que aportaron muy buenas sugerencias para mejorar el manuscrito. A las siguientes instituciones que financiaron los censos de lobos marinos en la Argentina por cuatro décadas: CONICET, ANPCyT, Wildlife Cons. Soc., Nat. Geographic Soc., Marineland Côte D’Azur, Prog. Coop. Cient. Iberamérica, PNUD, PNUMA, Fund. BBVA, Amnèville Zoo, Yaqu-Pacha, Heidelberg Zoo y Dutch Zoo. A las autoridades provinciales de Fauna y Áreas Protegidas de Río Negro, Chubut y Santa Cruz.

\section{REFERENCIAS}

Acevedo, J., A. Aguayo-Lobo, and W. Sielfeld. 2003. Eventos reproductivos del león marino común, Otaria flavescens (Shaw 1800), en el norte de Chile (Pacífico suroriental). Revista de Biología Marina y Oceanografía 38:69-75. https: //doi.org/10.4067/S0718-19572003000200003.

Anganuzzi, A. A. 1993. A comparison of tests detecting trends in abundance indices of dolphins. Fishery Bulletin 91: 183-194.

Aragones, L. V., T. A. Jefferson, and H. Marsh. 1997. Marine mammal survey techniques aplicable in developing countries. Asian Marine Biology 14:1539.

Bartheld, J. L., H. Pavés, F. Contreras, C. Vera, C. Manque, D. Miranda, D. Sepúlveda, P. Artacho, and L. Ossman. 2008. Cuantificación poblacional de lobos marinos en el litoral de la I a IV Región, Chile. Informe técnico Fondo de Investigación Pesquera IP-IT/2006-50. Chile.

Bastida, R., and D. Rodríguez. 2003. Mamíferos marinos de Patagonia y Antártida. Vazquez Mazzini. Buenos Aires. Argentina.

Baylis, A. M., R. A. Orben, J. P. Arnould, F. Christiansen, G. C. Hays, and I. J. Staniland. 2015. Disentangling the cause of a catastrophic population decline in a large marine mammal. Ecology 96:2834-2847. https://doi.org/10.1890/141948.1.

Bedriñana-Romano, L., F. A. Viddi, J. P. Torres-Florez, J. Ruiz, M. F. Nery, D. Haro, Y. Montecinos, and R. Hucke-Gaete. 2014. At-sea abundance and spatial distribution of South American sea lion (Otaria byronia) in Chilean Northern Patagonia: How many are there? Mammalian Biology 79:384-392. https://doi.org/10.1016/j.mambio.2014.07.002.

Berkson, J. M., and D. P. DeMaster. 1985. Use of Pup Counts in Indexing Population Changes in Pinnipeds. Canadian Journal of Fisheries and Aquatic Sciences 42:873-879. https://doi.org/10.1139/f85-111.

Buckland, S. T., and A. E. York. 2018. Abundance estimation. Pp. 1-6 in B. Würsig, J. G. M. Thewissen and K. M. Kovacs (eds.). Encyclopedia of Marine Mammals (3rd. ed.). Academic Press, London, UK.

Buckland, S. T., D. R. Anderson, K. P. Burnham, J. L. Laake, D. L. Borchers, and L. Thomas. 2001. Introduction to Distance Sampling: Estimating Abundance of Biological Populations. Oxford University Press, Oxford. England.

Campagna, C. 1985. The breeding cycle of the Southern sea lion, Otaria byronia. Marine Mammals Science 1:210-218. https://doi.org/10.1111/j.1748-7692.1985.tb00010.x.

Cárdenas-Alayza, S. 2018. South America sea lion (Otaria byronia). Pp. 907-910 in B. Würsing, J. G. M. Thewisseng and K. M. Kovacs (eds.). Encyclopedia of Marine mammals. Academic Press. https://doi.org/10.1016/B978-0-12-8043271.00238-7.

Cárdenas-Alayza, S., E. Crespo, and L. Oliveira. 2016. Otaria byronia. The IUCN red list of threatened species 2016 e.T41665A61948292. https://doi.org/10.2305/IUCN.UK.2016-1.RLTS.T4166 5A61948292.

Carrara, I. S. 1952. Lobos marinos, pingüinos y guaneras de las costas del litoral marítimo islas adyacentes de la República Argentina. Universidad Nacional de La Plata, Buenos Aires. Argentina.

Caughley, G. 1977. Analysis of Vertebrate Populations. John Wiley and Sons, Chichester, New York, Brisbane, Toronto. U.S.

Caughley, G., and A. Gunn. 1996. Conservation biology in theory and practice. Blackwell. Cambridge, U.S.

Consejo Federal Pesquero 2016. Plan de acción nacional para reducir la interacción de mamíferos marinos con pesquerías en la República Argentina 2015. 1a ed. Buenos Aires. Consejo Federal Pesquero. 
Contreras, F., J. Bartheld, M. Montecinos, F. Moreno, and J. Torres. 2014. Cuantificación poblacional de lobo marino común (Otaria flavescens) en el litoral de la XV, I y II Regiones. http://dx.doi.org/10.13140/RG.2.1.2497.0401.

Costello, M. J., M. Coll, R. Danovaro, P. Halpin, H. Ojaveer, and P. Miloslavich. 2010. A census of marine biodiversity knowledge, resources, and future challenges. PLOS ONE 5(8):e12110. https://doi.org/10.1371/journal.pone.0012110.

Crespo, E. A. 1988. Dinámica poblacional del lobo marino del sur Otaria flavescens (Shaw, 1800), en el norte del litoral patagónico. Tesis Doctoral. Universidad Nacional de Buenos Aires, Buenos Aires, Argentina. Pp. 298.

Crespo, E. A. 2021. Exploitation and Recovery of the South American Sea Lion in the Southwestern

Atlantic. Pp. 521-538 in Campagna C. and R. Harcourt (eds.). Ethology and Behavioral Ecology of Otariids and the Odobenid, Ethology and Behavioral Ecology of Marine Mammals. Springer.

https://doi.org/10.1007/978-3-030-59184-7.

Crespo, E. A., and S. N. Pedraza. 1991. Estado actual y tendencia de la población de lobos marinos de un pelo (Otaria flavescens) en el litoral norpatagónico. Ecología Austral 1:87-95.

Crespo, E. A., D. Oliva, S. Dans, and M. Sepúlveda. 2012. Estado de situación del lobo marino común en su área de distribución. Universidad de Valparaíso, Valparaíso. Chile.

Crespo, E. A., L. R. Oliveira, and M. Sepúlveda 2021. South American Sea Lion (Otaria flavescens, Shaw 1800). Pp. 93118 in Heckel G. and Y. Schramm (eds.). Ecology and Conservation of Pinnipeds in Latin America. Springer. https: //doi.org/10.1007/978-3-030-63177-2

Dans, S. L., E. A. Crespo, S. N. Pedraza, and M. Koen Alonso. 2004. Recovery of the South American sea lion population in northern Patagonia. Canadian Journal of Fishery and Aquatic Science 61:1681-1690. https://doi.org/10.1139/f04-105.

Eberhardt, L. L., D. G. Chaphan, and J. R. Gilbert. 1979. A review of marine mammal census methods. Wildlife Monographs 63:3-46.

Feijoo, M., E. P. Lessa, R. Loizaga de Castro, and E. A. Crespo. 2011. Mitochondrial and microsatellite assessment of population structure of South American sea lion (Otaria flavescens) in the Southwestern Atlantic Ocean. Marine Biology 158(8):1857-1867. https://doi.org/10.1007/s00227-011-1697-4.

Forney, K. A. 2000. Environmental models of cetacean abundance: Reducing uncertainty in population trends. Conservation Biology 14:1271-1286. https://doi.org/10.1046/j.1523-1739.2000.99412.x.

Franco-Trecu, V., M. Drago, C. Baladán, M. D. García-Olazábal, E. A. Crespo, L. Cardona, and P. Inchausti. 2015. Postharvesting population dynamics of the South American sea lion (Otaria byronia) in the southwestern Atlantic. Marine Mammal Science 31:963-978. https://doi.org/10.1111/mms.12197.

Franco-Trecu, V., M. Drago, M. F. Grandi, A. Soutullo, E. A. Crespo, and P. Inchausti. 2019. Abundance and Population Trends of the South American Fur Seal (Arctocephalus australis) in the Southern Atlantic. Aquatic Mammals 45:48-55. https://doi.org/10.1578/AM.45.1.2019.48.

Garner, G. W., S. C. Amstrup, J. L. Laake, B. F. J. Manly, L. L. Mcdonald, and D. G. Robertson (eds.). 1999. Marine mammal survey and assessment methods. A. A. Balkema, 3000 BR Rotterdam, Netherlands. Pp. 287.

Giardino, G. 2014. Estructura y dinámica de las colonias de lobos marinos de un pelo de la Provincia de Buenos Aires, y su relación con pesquerías de la región. Tesis Doctoral. Universidad Nacional de Mar del Plata, Buenos Aires, Argentina. Pp. 173.

Giardino, G. V., M. A. Mandiola, J. Bastida, P. E. Denuncio, R. O. Bastida, and D. H. Rodríguez. 2016. Travel for sex: Long-range breeding dispersal and winter haulout fidelity in southern sea lion males. Mammalian Biology 81:89-95. https://doi.org/10.1016/j.mambio.2014.12.003.

Godoy, J. C. 1963. Fauna Silvestre. Consejo Federal de Inversiones, Argentina.

Grandi, M. F. 2010. Dinámica poblacional y cambios estructurales en una población en crecimiento de lobo marino común, Otaria flavescens, del litoral norpatagónico. Tesis Doctoral. Universidad Nacional del Comahue, Bariloche, Argentina. Pp. 197.

Grandi, M. F., S. L. Dans, and E. A. Crespo. 2008. Social composition and spatial distribution of colonies in an expanding population of South American sea lions. Journal of Mammalogy 89:1218-1228. https://doi.org/10.1644/08-MAMMA-088.1.

Grandi, M. F., S. L. Dans, and E. A. Crespo. 2015. The recovery process of a population is not always the same: the case of Otaria flavescens. Marine Biology Research 11:225-235. https://doi.org/10.1080/17451000.2014.932912.

Grandi, M. F., R. Loizaga de Castro, E. Terán, M. R. Santos, G. Bailliet, and E. A. Crespo. 2018. Is recolonization pattern related to female philopatry? An insight into a colonially breeding mammal. Mammalian Biology 89:21-29. https: //doi.org/10.1016/j.mambio.2017.12.002.

Hoffman, J. I., G. J. Kowalski, A. Klimova, L. J. Eberhart-Phillips, I. J. Staniland, and A. M. M. Baylis. 2016. Population structure and historical demography of South American sea lions provide insights into the catastrophic decline of a marine mammal population. Royal Society Open Science 3:160291. https://doi.org/10.1098/rsos.160291.

Johnston, D. W., J. Dale, K. T. Murray, E. Josephson, E. Newton, and S. Wood. 2017. Comparing occupied and unoccupied aircraft surveys of wildlife populations: assessing the gray seal (Halichoerus grypus) breeding colony on Muskeget Island, USA. Journal of Unmanned Vehicle Systems 5(4):178-191. https://doi.org/10.1139/juvs-2017-0012.

Lewis, M. N., and I. Ximénez. 1983. Dinámica de la población de Otaria flavescens (Shaw) en el área de Península Valdés y zonas adyacentes (segunda parte). Contribución 79. Centro Nacional Patagónico, Puerto Madryn, Chubut, Argentina.

Lonergan, M., D. Thompson, L. Thomas, and C. Duck. 2011. An approximate Bayesian method applied to estimating the trajectories of four British grey seal (Halichoerus grypus) populations from pup counts. Journal of Marine Science Vol. 2011. Article ID 597424, 7 pages. https://doi.org/10.1155/2011/597424.

Lowry, M. S. 1999. Counts of California sea lion (Zalophus californianus) pups from aerial color photographs and from the ground: A comparison of two methods. Marine Mammal Science 15(1):143-158. https://doi.org/10.1111/j.17487692.1999.tb00786.x

Mandiola, A., G. Giardino, R. Bastida, and D. Rodríguez. 2010. Variación espacio-temporal en la distribución del lobo marino de un pelo (Otaria flavescens) en el puerto Mar del Plata (Argentina) durante los últimos 30 años. XIV Reunião de Trabalho de Especialistas em Mamíferos Aquáticos da América do Sul (RT) $8^{\circ}$ Congresso da Sociedade 
Latinoamericana de Especialistas em Mamíferos Aquáticos (SOLAMAC).

McIntosh, R. R., R. Holmberg, and P. Dann. 2018. Looking without landing-using remote piloted aircraft to monitor fur seal populations without disturbance. Frontiers in Marine Science 5:202. https://doi.org/10.3389/fmars.2018.00202.

Milano, V. N., M. F. Grandi, A. C. M. Schiavini, and E. A. Crespo. 2020. Sea lions (Otaria flavescens) from the end of the world: insights of a recovery. Polar Biology 43:695-706. https://doi.org/10.1007/s00300-020-02672-9.

Oliva, D., W. Sielfeld, M. Buscaglia, M. Matamala, R. Moraga, H. Pavés, M. J. Pérez, D. Schrader, M. Sepúlveda, and A. Urra. 2008. Plan de acción para disminuir y mitigar los efectos de las interacciones del lobo marino común (Otaria flavescens) con las actividades de pesca y acuicultura. Fondo de Investigación Pesquera, Informe Técnico IP-IT/200634. Chile.

Oliva, D., M. Sepúlveda, L. R. Durán, A. Urra, W. Sielfeld, R. Moraga, G. Pavés, and L. Muñoz. 2012. Cuantificación poblacional de lobos marinos en las Regiones X-XI y propuesta de escenarios de manejo. Informe Final Proyecto FAP ID 4728-46LP11. Chile.

Oliva Ekelund, D., L. R. Durán Figueroa, D. Cárcamo Segovia, M. Pizarro, M. Sepúlveda Martínez, C. Anguita, A. Canto, P. Herrera, L. Muñoz, M. Orellana, M. Santos, and P. Vásquez. 2020. Estimación poblacional de lobos marinos e impacto de la captura incidental. Informe Final Proyecto FIP 2018-54. Chile. URL: tinyurl.com/5zn4f4.

Oliveira, L. R., and P. Majluf. 2012. Recuperación de la población de lobos de un pelo después del más fuerte evento de El Niño en la historia. Pp. 12 en E. A. Crespo, D. Oliva, S. Dans and M. Sepúlveda (eds.). Estado de situación del lobo marino común en su área de distribución (Universidad de Valparaíso, Valparaíso. Chile.

Oliveira, L. R., M. C. Gehara, L. D. Fraga, F. Lopes, J. I. Túnez, M. H. Cassini, P. Majluf, S. Cárdenas-Alayza, H. J. Pavés, E. A. Crespo, N. García, R. Loizaga de Castro, A. R. Hoelzel, M. Sepúlveda, C. Olavarría, V. H. Valiati, R. Quiñones, M. J. Pérez-Alvarez, P. H. Ott, and S. L. Bonatto. 2017. Ancient female philopatry, asymmetric male gene flow, and synchronous population expansion support the influence of climatic oscillations on the evolution of South American sea lion (Otaria flavescens). PLoS One 12(6):e0179442. https://doi.org/10.1371/journal.pone.0179442.

Páez, E. 2006. Situación de la administración del recurso lobos y leones marinos en Uruguay. Pp. 577-583 en R. Menafra, L. Rodríguez-Gallego, F. Scarabino and D. Conde (eds.). Bases para la conservación y el manejo de la costa Uruguaya. Vida Silvestre, Sociedad Uruguaya para la Conservación de la Naturaleza. Montevideo, Uruguay.

Pavanato, H., K. G. Silva, S. C. Estima, D. S. Monteiro, and P. G. Kinas. 2013. Occupancy dynamics of South American sea-lions in Brazilian haul-outs. Brazilian Journal of Biology 73(4):855-862. https://doi.org/10.1590/S151969842013000400023.

Pavés, H. J., R. P. Schlatter, and C. I. Espinoza. 2005. Patrones reproductivos del lobo marino común, Otaria flavescens (Shaw 1800), en el centro-sur de Chile. Revista Chilena de Historia Natural 78:687-700. https://doi.org/10.4067/S0716078X2005000400008.

Payne, M. R. 1978. Population size and age determination in the Antarctic Fur seal Arctocephalus gazella. Mammal Review 8:67-73. https://doi.org/10.1111/j.1365-2907.1978.tb00218.x.

Payne, J. L., A. M. Bush, N. A. Heim, M. L. Knope, and D. J. McCauley. 2016. Ecological selectivity of the emerging mass extinction in the oceans. Science 353(6305):1284-1286. https://doi.org/10.1126/science.aaf2416.

QGIS Development Team. 2019. QGIS Geographic Information System. Open Source Geospatial Foundation. URL: qgis.org.

Raoult, V., A. P. Colefax, B. M. Allan, D. Cagnazzi, N. Castelblanco-Martínez, D. Ierodiaconou, D. W. Johnston, S. Landeo-Yauri, M. Lyons, V. Pirotta, G. Schofield, and P. A. Butcher. 2020. Operational Protocols for the Use of Drones in Marine Animal Research. Drones 4(4):64. https://doi.org/10.3390/drones4040064.

Rasband, W. S. 2013. ImageJ, U. S. National Institutes of Health, Bethesda, Maryland, USA. URL: imagej.nih.gov/ij.

Reyes, L., and E. A. Crespo. 1994. Fluctuaciones diarias y lunares en el número de lobos de un pelo en agrupaciones no reproductivas. Proceedings $4^{\circ}$ Reunión de Trabajo de Especialistas en Mamíferos Acuáticos. América del Sur, 12-15 Nov. 1990. Valdivia, Chile. Pp. 212-226.

Reyes, L. M., E. A. Crespo, and V. Szapkievich. 1999. Distribution and population size of the southern sea lion (Otaria flavescens) in central and southern Chubut, Argentina. Marine Mammal Science 15:478-493. https://doi.org/10.1111/ j.1748-7692.1999.tb00814.x.

Riedman, M. 1990. The pinnipeds; seals, sea lions, and walruses. University California Press, Berkeley. U.S. https: //doi.org/10.1525/9780520320086.

Romero, M. A., M. F. Grandi, M. Koen-Alonso, G. Svendsen, M. O. Reinaldo, N. A. García, S. L. Dans, R. González, and E. A. Crespo. 2017. Analysing the natural population growth of a large marine mammal after a depletive harvest. Scientific Reports 7(1):1-16. https://doi.org/10.1038/s41598-017-05577-6.

Rosas, F. C. W., M. C. Pinedo, M. Marmontel, and D. M. Haimovici. 1994. Seasonal movements of the South American sea lion (Otaria 'avescens, Shaw) off the Rio Grande do Sul coast, Brazil. Mammalia 58:51-59. https://doi.org/10.1515/ mamm.1994.58.1.51.

Rueda, N. B., R. A. Arakaki, and J. C. Fano. 2020. Uso de Aeronaves Remotamente Pilotadas (RPA) en la estimación de poblaciones de lobos marinos en la Isla Chincha sur-Ica. Industrial Data 23(2):7-19. https://doi.org/10.15381/ idata.v23i2.16921.

Rueden, C. T., J. Schindelin, M. C. Hiner, B. E. DeZonia, A. E. Walter, E. T. Arena, and K. W. Eliceiri. 2017. ImageJ2: ImageJ for the next generation of scientific image data. BMC Bioinformatics 18:529. https://doi.org/10.1186/s12859017-1934-z.

Schiavini, A. C. M., E. A. Crespo, and V. Szapkievich. 2004. Status of the population of South American sea lion (Otaria favescens) in Santa Cruz and Tierra del Fuego Provinces, Argentina. Mammalian Biology 69:1-11. https://doi.org/ 10.1078/1616-5047-00125.

Sepúlveda, M., D. P. Oliva, and F. J. Palma. 2001. Daily and annual circarhythms activity in the South American sea lion Otaria flavescens (Carnivora: Otariidae) at the central zone of Chile. Revista de Biología marina y Oceanografía 36(2):181-188. https://doi.org/10.4067/S0718-19572001000200006.

Sepúlveda, M., P. Inostroza, M. J. Pérez-Álvarez, D. Oliva, and R. Moraga. 2009. Seasonal variation in the abundance of South American sea lions Otaria flavescens (Shaw, 1800) in Chañaral Island, Reserva Nacional Pingüino de Humboldt, Chile. Revista de Biología Marina y Oceanografía 44(3):685-689. https://doi.org/10.4067/S0718-19572009000300014. 
Sepúlveda, M., D. Oliva, A. Urra, M. J. Pérez-Álvarez, R. Moraga, D., Schrader, P. Inostroza, A. Melo, H. Díaz, and W. Sielfeld. 2011. Distribution and abundance of the South American sea lion Otaria flavescens (Carnivora: Otariidae) along the central coast off Chile. Revista Chilena de Historia Natural 84:97-106. https://doi.org/10.4067/S0716078X2011000100007.

Sepúlveda, M., R. A. Quiñones, P. Carrasco, and M. J. Pérez-Álvarez. 2012. Daily and seasonal variation in the haul-out behavior of the South American sea lion. Mammalian Biology 77(4):288-292. https://doi.org/10.1016/ j.mambio.2012.03.008.

Sepúlveda, M., M. Santos, R. Veas, L. Muñoz, D. Olea, R. Moraga, and W. Sielfeld. 2015. Variaciones anuales, estacionales y diarias en la abundancia del lobo marino común Otaria flavescens en dos colonias reproductivas del norte de Chile. Revista de Biología Marina y Oceanografía 50(2):205-220. https://doi.org/10.4067/S0718-19572015000300001.

Teilmann, J., F. Rigét, and T. Harkonen. 2010. Optimizing survey design for Scandinavian harbour seals: population trend as an ecological quality element. ICES Journal of Marine Science 67:952-958. https://doi.org/10.1093/icesjms/fsq001.

Túnez, J. I., D. Centrón, H. L. Cappozzo, and M. H. Cassini. 2007. Geographic distribution and diversity of mitochondrial DNA haplotypes in South American sea lions (Otaria flavescens) and fur seals (Arctocephalus australis). Mammalian biology 72(4):193-203.

Túnez, J. I., H. L. Cappozzo, M. Nardelli, and M. H. Cassini. 2010. Population genetic structure and historical population dynamics of the South American sea lion, Otaria flavescens, in north-central Patagonia. Genetica 138(8):831-841. https: //doi.org/10.1007/s10709-010-9466-8.

Udevitz, M. S., C. Jay, and M. Cody. 2005. Observer variability in pinniped counts: Ground-based enumeration of walruses at haul-out sites. Marine Mammal Science 21:108-120. https://doi.org/10.1111/j.1748-7692.2005.tb01211.x.

Venegas, C., J. Gibbons, A. Aguayo, W. Sielfeld, J. Acevedo, N. Amado, J. Capella, G. Guzmán, and C. Valenzuela. 2002. Distribución y abundancia de lobos marinos (Pinnipedia: Otariidae) en la Región de Magallanes. Anales del Instituto de la Patagonia, Serie Ciencias Naturales 30:67-82.

Weinberger, C. 2013. El lobo marino común, Otaria flavescens, en Chile: distribución espacial, historia demográfica y estructuración genética. Tesis Doctoral. Pontificia Universidad Católica de Chile.

Wickens, P., and P. A. Shelton. 1992. Seal pup counts as indicators of population size. South African Journal of Wildlife Research 22:65-69.

Wright, S. J. 2005. A photographic atlas of developmental biology. Englewood, IL: Morton Publishing Company.

Ximénez, I. 1975. Dinámica de la población de Otaria flavescens (Shaw) en el área de Península Valdés y zonas adyacentes (Provincia del Chubut, RA). Centro Nacional Patagónico, Puerto Madryn, Chubut, Argentina. Informe Técnico 1.4.1: $1-52$.

York, A. E. 1987. On comparing the population dynamics of fur seals. Pp. 1-212 in J. P. Croxall and R. L. Gentry (eds.). Status, biology, and ecology of fur seals. NOAA Technical Report NMFS 51. U.S. 